\title{
Die Nord-Süd-Globalverhandlungen : Rueckblick, neue Runde und der Beitrag der Schweiz
}

Les négociations globales : rétrospective, nouvelle étape du dialogue Nord-Sud et la contribution de la Suisse

\section{Beat-Alexander Jenny}

\section{OpenEdition}

\section{Journals}

Electronic version

URL: http://journals.openedition.org/sjep/922

ISSN: 1663-9677

\section{Publisher}

Institut de hautes études internationales et du développement

\section{Printed version}

Date of publication: 1 janvier 1981

Number of pages: 181-211

ISSN: $1660-5926$

\section{Electronic reference}

Beat-Alexander Jenny, « Die Nord-Süd-Globalverhandlungen : Rueckblick, neue Runde und der Beitrag der Schweiz », Schweizerisches Jahrbuch für Entwicklungspolitik [Online], 1 | 1981, Online erschienen am: 27 Mai 2013, abgerufen am 07 September 2020. URL : http://journals.openedition.org/sjep/922 


\title{
DIE NORD-SÜD-GLOBALVERHANDLUNGEN : RUECKBLICK, NEUE RUNDE UND DER BEITRAG DER SCHWEIZ
}

\author{
Beat-Alexander Jenny
}

\begin{abstract}
LES NEGOCIATIONS GLOBALES : RETROSPECTIVE, NOUVELLE ETAPE DU DIALOGUE NORD-SUD ET LA CONTRIBUTION DE LA SUISSE
\end{abstract}

\begin{abstract}
Résumé
Après un rappel des principales étapes du processus qui a mené au dialogue Nord-Sud, l'auteur s'interroge sur les causes de l'insuccès et sur les obstacles à la poursuite de ce programme. En particulier il analyse les difficultés qui entravent l'adoption, par les Nations Unies, d'un projet définissant les modalités d'un nouveau round de négociations globales. L'attitude de la Suisse, à l'égard de ce projet, est mise en évidence. Les conclusions, sur le même sujet, de la Conférence d'Ottawa, sont aussi objet de réflexion. Pour terminer, l'auteur évoque les tendances actuelles de la politique de la Confédération en matière d'aide au développement et suggère des mesures qui permettraient de renforcer cette politique sans qu'il y ait nécessairement augmentation des dépenses publiques.
\end{abstract}

\section{VORGESCHICHTE}

Das wachsende Selbstbewusstsein der Staaten der dritten Welt und die fröhen Versuche zur Globalisierung ihrer Anliegen können erstmals in den 1960er Jahren festgestellt werden. Wahrend der Vorbereitungen der ersten UNO-Konferenz Uber Handel und Entwicklung (UNCTAD 1), im Jahre 1963, schlossen sich 75 (ein Jahr spater 77) Entwicklungslander angesichts der wachsenden Divergenzen und Interessenkonflikte zwischen Industrieländern und der dritten Welt (namentlich auf den Gebieten der Rohstoffe und der Struktur des Welthandels) erstmals zu einer gemeinsamen Absichtserklärung und Verhandlungsstrategie zusammen.

Nach der Errichtung der UNCTAD als ständiges Organ der UNOGeneralversammlung bildete sich die Gruppe der 77 (deren Mitgliederzahl stetig anschwoll und heute 122 Lander zohlt) zum Sprachrohr der dritten Welt, ja zum entscheidenden Verhandlungspartner in 
allen UNO-Organen und -Organisationen heran.

Bis zum Ende der 1960er Jahre befanden sich die Industrielunder in einer noch zufriedenstellenden Epoche wirtschaftlichen Wachstums und expandierenden Welthandels. Die Volkswirtschaften der Entwicklungslander hatten wenig Gewicht im internationalen Geschehen, und Industrielander zählten für ihre Produkte und Märkte grosso modo wenig auf Entwicklungslander. Wohl kam es gelegentlich vor, dass Industrielander und transnationale Gesellschaften von spezifischen Rohstoffen aus einzelnen Entwicklungsländern in Abhängigkeit gerieten, aber angesichts der ungleichen wirtschaftlichen und politischen Gewichte der fraglichen Lünder wurden diebezllgliche Probleme, wenn sie entstanden, gelठtst bevor sie auf der Ebene globaler Verhandlungen in Erscheinung treten konnten.

Auf globaler Ebene reduzierten sich die "Verhandlungen" bis etwa zur UNCTAD III (1972) auf ein Aufstellen von Forderungskatalogen seitens der Entwicklungslünder und ein unendliches Zerreden solcher Forderungen seitens der Industrielander, wobei am Ende gelegentlich einige Brosamen (wie Zugestandnisse im Rahmen des GATT oder bezÜglich der Offentlichen Entwicklungshilfe) an die dritte Welt verteilt werden konnten. Die Industrieländer glaubten zwar an den allgemeinen Nutzen eines globalen Dialoges Bber Entwicklung und Welthandel, konnten sich jedoch kaum ernsthafte Probleme vorstellen, die aus der dritten Welt kamen und welche Lossungen in der Form von globalen Verhandlungen bedurften.

Aber die weltwirtschaftlichen Zusammenhänge underten sich drastisch und grundsatzlich in den frühen 1970er Jahren, haupts der durch die USA ausgelbsten Währungskrise, der wachsenden Inflation in den Industrielandern und der Hochkonjunktur auf den Rohstoffmärkten. Hohe Preise für Rohstoffe wie Zucker, Kupfer, Bauxit und Kaffee vermochten das politische Bewusstsein vieler EntwicklungsIander zu verstärken - eine Tatsache, die sich in den entsprechenden Rohstoffverhandlungen im Rahmen der UNCTAD widerspiegelte. Für die dritte Welt war die Möglichkeit eines Griffes an die Hebel der Interdependenz, durch kollektives Ausüben ihrer Rohstoffmacht, in die Nahe gerückt.

Als Wendepunkt mag der Herbst 1973 angenommen werden, angefangen mit der vierten Gipfelkonferenz der blockfreien Staaten in Algier 
(September) und fortgeführt mit dem ersten Beschluss der OPECStaaten, den Erdblpreis unilateral zu erhbhen (Oktober). In Algier beschlossen die blockfreien Staaten im Rahmen der UNO gemeinsam zu handeln und den für die Entwicklung der dritten Welt als notwendig empfundenen weltwirtschaftlichen Strukturwandel herbeizuführen. Mit dem Erd8l als Trumpfkarte schien die Verwirklichung dieser politischen Initiative nicht mehr so weit hergeholt zu sein wie im vergangenen Jahrzehnt.

Eine Wende bahnte sich auch im Inhalt der Entwicklungspolitik und in der Wirtschaftspolitik Uberhaupt an. DiesbezÜgliche Signale und Impulse kamen schon von der globalen Umweltschutzkonferenz in Stockholm (1972). In den Industrielandern ging das seit 1945 ununterbrochene Wachstumsdenken, das auch die Beziehungen zur dritten Welt geprolgt hatte, seinem Ende zu - und dies allein schon aus wirtschaftlichen Gründen : Wirtschaftswachstum allein brachte nicht mehr Vollbeschaftigung, dafur aber zunehmende negative Begleiterscheinungen (soziale Konflikte, Zerstorung der Natur und des Habitat); im Ubrigen meldeten sich Impulse für den Strukturwandel aus dem wachsenden Kostendruck in der Industrie sowie aus der zunehmenden Verunsicherung im internationalen Wührungssystem. Sollte es moglich sein, dass der unvermeidbare Strukturwandel in den Indutrielündern in einer Weise vollzogen wörde, der auch den Entwicklungslündern nützlich wäre - ja deren Entwicklungsspielraum und deren Eigenständigkeit zu vergrbssern vermbchte ?

\section{DIE ERSTE RUNDE}

In den Jahren von 1974 bis 1977 entfaltete sich Uber dieses Thema eine rege internationale Konferenztltigkeit, in welcher der Versuch zur Globalisierung der Problematik der Entwicklung und der internationalen Wirtschaftsbeziehungen unter vielen verschiedenen Blickpunkten unternommen wurde - zum Beispiel im Rahmen des GATT (1975 ff.), der UNCTAD IV (1976), der Weltbeschaftigungskonferenz (1976) und in verschiedenen Welternathrungsgespratchen (1974-77). Den Grundton haben die blockfreien Staaten in der sechsten (1974) und siebten (1975) Sondersession der UNO-Generalversammlung, 
unter der Führung Algeriens, angeschlagen : es wurde eine de-facto Fusion der Blockfreien und der Gruppe der 77 angestrebt, wobei die erstere die "politische Partei" und die letztere die "Gewerkschaft" der dritten Welt bilden sollte. Dank der durch die neue weltwirtschaftliche Situation entstandenen Verhandlungsbereitschaft der Industrielunder gelang es, im Konsensusverfahren die Begründung einer neuen Weltwirtschaftsordnung festzulegen. Unter Bedenken seitens einiger Industrieländer wurde ferner die Charta der wirtschaftlichen Pflichten und Rechte der Staaten formuliert und zu verabschiedet. Seither haben die Staaten der dritten Welt in allen Nord-SUd-Gesprachen und Verhandlungen auf die Durchfürung der in diesen Grundtexten ausgesprochenen Absicht beharrt.

Die westlichen Industrielander haben ihrerseits versucht, die Initiative im weltwirtschalflichen Geschehen zurb̈ckzugewinnen. Der Pariser Nord-SUd-Dialog (KIWZ) entsprang einem Vorschlag des franzobsischen Staatspräsidenten, der im Herbst 1974 die OPECStaaten zu einem "Energiedialog" einzuladen versuchte. Im Vordergrund hatte die Versorgung und Preisbildung im Erdblsektor stehen sollen. Weshalb hatten die OPEC-Staaten jedoch an einem Energiedialog interessiert sein sollen? Unter AusnÜtzung der Uneinigkeit unter den Industrieländern und gestützt auf das neue Gefühl der Identitat und der Macht der Drittweltstaaten, hielten die OPECStaaten an der neugeschmiedeten blockfreien Solidaritat fest. Sie verlangten globale Gespräche, denn die Fragen der Energie und der Entwicklung waren ihrer Meinung nach miteinander verkn Upft : Mögliche Zugeständnisse seitens der OPEC-Staaten auf dem ErdరIsektor mÜsten direkt auf Zugeständnisse der Industrielünder in der Frage der Durchführung der neuen Weltwirtschaftsordnung abgestÜtz† werden.

Diese Formel Uffnete, vom Standpunkt der dritten Welt, die TÜr zu ernsthaften Verhandlungen. In Paris einigte man sich auf vier globale Themenbereiche : Rohstoffe, Energie, Entwicklung und Finanz. Der Pariser Dialog fand ausserhalb der UNO statt und war (unter Ausschluss u.a. der sozialistischen Industrielander Osteuropas) auf 27 Mitglieder beschrankt - 19 Lünder der Gruppe der 77 und 8 Delegationen aus dem Norden, 7 Industrielander (unter ihnen die Schweiz) plus die Europatische Gemeinschaft, die kollektiv vertreten war. LosgelUst von der UNO, frei vom Ublichen Konferenzge- 
baren und ohne ein omniprasentes Sekretariat erhoffte man, mit einer kleinen Anzahl wichtiger Lunder, durch die Nord-SUd-Rhetorik durchzudringen. Man wollte mehr erreichen - ja sogar zu pragmatischen Resultaten kommen und (seitens der Industrieländer) mit so wenig wie möglich Zugeständnissen in der Energiekrise wieder die Oberhand gewinnen.

Die Schweiz, die als Nicht-Mitglied der UNO die Globalverhandlungen und die entwicklungspolitischen Erneuerungen der frobhen 1970er Jahre als passiver Zuschauer verfolgte, fand Gefallen am Konzept sowie am kleinen Rahmen der Pariser KIWZ. Sie verstand es, die Ubrigen OECD-Lander (unter Hinweis auf ihre aussen- und kapitalwirtschaftliche Bedeutung) zu Uberzeugen, unter die Pariser Acht aufgenommen zu werden - auf Kosten Oesterreichs, des anderen neutralen Kleinstaates, der draussen bleiben musste.

Der Pariser Dialog führte jedoch zu keinem globalen Resultat. Nach fast zwei Jahren, unter stetiger "UNCTAD-isierung" der Verhandlungen, wurde weder auf dem Energiesektor noch in dem von den Entwicklungslandern geforderten Strukturwandel im Rahmen der neuen Weltwirtschaftsordnung ein Durchbruch erzielt. Der bescheidene Schlussbericht wurde mit einem unfertigen Anhang Uber die Verhandlungen in den vier Themenbereichen an die UNOGeneralversammlung zur Kenntnisnahme weitergeleitet. Die Fronten hatten sich namentlich unter dem Einfluss der USA verhartet. Niemand hat seither eine Weiterfllhrung der Pariser-Formel des NordSUd Dialoges ernsthaft wieder in Erwägung gezogen.

\section{DER STILLSTAND DES NORD-SÜD DIALOGES}

Die Gründe för dieses Versagen und für den Mangel an Fortschritt in anderen globalen Konferenzen, sind verschiedenen gelagert und sind, wie das bei den meisten Themen der Nord-SUd-Problematik der Fall ist, komplex. Einige sollen hier erwähnt werden, um zu versuchen, eine Verständnisgrundlage für den aktuellen, neuen Ansatz zu Globalverhandlungen zu schaffen.

Erstens gehort es zwar zum Verhandlungsgebaren der dritten Welt, 
keine Initiative ausser Acht zu lassen, die Gehb̈r für ihre Anliegen und Forderungen zu verschaffen vermag. Dies erklürt in erster Linie ihr Engagement in der Pariser Formel. Aber im Grunde ziehen die Lander der dritten Welt die UNO als Verhandlungsforum allen anderen Mタglichkeiten, wie dem internationalen Wuhrungsfonds, der Welt Bank, dem GATT und thnlichen nicht-universellen oder rein technisch orientierten Korperschaften vor. Sie legen mehr Wert auf grundsätzliche Verständigung und Abkommen auf politischer Ebene in der UNO-Generalversammlung, als auf Verhandlungen in technischen Gremien, die - obwohl wichtig zur Ausarbeitung von Einzelheiten - als zweitrangig eingestuft werden. Sogar auf technischer Ebene, wie bei den Verhandlungen zur Vorbereitung des gemeinsamen Rohstoffonds, wurde der UNO der Vorzug gegeben, weil das System des Stimmrechtes bei anderen Gremien (wie bei der Welt Bank und dem Wahrungsfonds) zu Ungunsten der dritten Welt gewichtet ist.

Zweitens war spattestens 1977 im Nord-SUd-Verhandlungsklima wieder ein Wandel eingetreten. Der durch die Energiekrise ausgelbste Druck auf die Industrielunder, der zuvor eine gewisse Bereitschaft zu Konzessionen hervorger fen hatte, liess nach. Die erste Erdolkrise war mehr oder weniger gebundigt, der Kreislauf der OPEC-UeberschUsse in Überschaubare und för die Industrielander vorteilhafte Bahnen geleitet. Zuversicht bestand auf die Nutzbarmachung never Energiequellen ausserhalb des OPEC-Bereiches. Im Ubrigen waren die Ueberschüsse der OPEC-Lünder, wie deren Führungsrolle im Rahmen der Gruppe der 77, angesichts neuer wirtschaftlicher Verflechtungen mit dem industrialisierten Westen im Schwinden begriffen. Die Hoffnung auf den Schritt der OPEC-Staaten vom Bekenntnis zur blockfreien Solidaritüt, zur wirtschaftlichen Allianz und Verknß̈pfung mit der Ubrigen dritten Welt erschien verfrüht oder verschoben.

Drittens sahen sich die Industrieländer nach der UNCTAD IV (1976) und nach dem Abschluss der Pariser Gespräche mehr und mehr gezwungen, ihre eigene Rezession ernst zu nehmen und vornehmlich untereinander Gespräche zu führen - sei es im Rahmen der Währungsproblematik oder in den multilateralen Handelsgesprachen. Obwohl die Problematik der dritten Welt nach dieser Gewichtsverlagerung der Prioritlten weiterhin eine gewisse Rolle gespielt haben mochte, fanden keine wirklichen Nord-Süd Verhandlungen mehr statt, in 
welchen der globale Strukturwandel im Mittelpunkt verblieben wäre. Viertens scheinen die Verhandlungsstrategie und der innere Zusammenhang der Gruppe der 77 in den Jahren nach 1977 an Kraft verloren zu haben. Dieses Phanomen kann an der Rohstoffdebatte veranschaulicht werden. Seit der UNCTAD IV (1976) steht die Preisstabilisierung der Rohstoffe durch Intervention in den Märkten im Vordergrung der Verhandlungen. Der Rahmen dazu soll durch das integrierte Rohstoffprogramm und den gemeinsamen Rohstofffonds gebildet werden. Zwar enthielt die entsprechende UNCTADResolution 93 (IV) mehr als bloss das Gebot der Preisstabilisierung : es ist die Rede von der Erhaltung der gesamten Exporteinnahmen der Entwicklungslander, von der Verarbeitung der Rohstoffe in den Produzentenlandern, von der Diversifizierung der Exporte der Rohstofflander, Erhaltung der Wettbewerbsfahigkeit von Natur produkten und von anderem wie Zugang zu Mürkten und Bekumpfung von Handelsschranken. Marktintervention und internationale Preispolitik waren jedoch das Instrumentarium, das die dritte Welt kollektiv am meisten zu mobilisieren vermochte. Man stellte sich vor, dass Produzenten- und Konsumentenlunder gemeinsam für alle im integrierten Programm enthaltenen Rohstoffabkommen Reservelager anlegen wÜrden, welche mit Hilfe des gemeinsamen Rohstoffonds zur Erhaltung der Preisstabilitat strategisch eingesetzt werden kbnnen. Die ziemlich einseitige und starre Konzentration der dritten Welt auf dieses Interventionsinstrumentarium mag auch in der Hoffnung begrundet gewesen sein, den Erfolg der OPEC-Staaten in der Kontrolle des ErdBlmarktes in bescheidenem Masse in anderen Murkten zu wiederholen. Nach vier Jahren Verhandlungen ist das Resultat jedoch ausserst bescheiden ausgefallen :

1. Im Juni 1980 sind die Statuten des gemeinsamen Rohstoffonds zu Ende diskutiert und von der Verhandlungskonferenz angenommen worden; der Fonds selber ist gegenüber dem ursprünglichen Vorschlag (US \$ $6 \mathrm{mia}$ ) erheblich reduziert worden (zwei Hauptkonten mit total US $\$ 750 \mathrm{mio}$ ) und enthalt keinen Zeitplan zur Wiederaufstockung;

2. Den bereits bestehenden vier Rohstoffabkommen des integrierten Programmes (Kaffee, Kakao, Zinn und Zucker) ist bloss ein neues Abkommen (Kautschuk) hinzugefügt, und ein bestehendes Abkommen (Zucker) neuverhandelt worden. Die Ubrigen 13 Rohstoffverhandlungen des integrierten Programmes sind entweder noch in einiger 
Ferne eines Abschlusses oder haben noch gar nicht begonnen.

Als Gründe für diesen mageren Fortschritt werden von Spezialisten in den Industrieländern gelegentlich Mängel im Bkonomischen Konzept ins Feld geführt. Diese, wenn sie zutreffen, sind jedoch sekundarrer Natur. Der Hauptgrund besteht, wie das bei den anderen globalen Verhandlungsthemen auch der Fall ist, darin, dass die Lünder der dritten Welt zwar ohne grosse Schwierigkeiten einem politischen Konzept und einer allgemeinen Zielsetzung zustimmen können, dass aber ihre Solidaritat und Verhandlungstaktik Auflbsungserscheinungen zeigen, wenn es um das Verhandeln und Durchsetzen von konkreten technischen Einzelheiten der globalen Vertragswerke geht.

Dass derartige Voraussetzungen vornehmlich den Industrie- und Ubrigen Ueberschussländern nützen und gar nicht zu ernsthaften Verhandlungen führen kBnnen, ist im Kreise der Gruppe der 77 wohl bekannt, doch hat diese Erkenntnis noch nicht zu einer Revision der Verhandlungstaktik geführt.

Als Endstation dieser Phase des Zerfalles und des Versagens kOnnen die fünfte UNCTAD (Mai 1979), in welcher die Verhandlungen Uber den globalen Strukturwandel auswegslos blockiert waren, und die dritte UNIDO-Generalkonferenz (Januar/Februar 1980), in welcher uberhaupt keine Schlussvereinbarung erreicht werden konnte, betrachte werden.

\section{DER NEUE ANSATZ ZU GLOBALVERHANDLUNGEN}

Der neve, heute aktuelle Ansatz zu Globalverhandlungen entstammt wiederum dem Lager der blockfreien Staaten. Er wurde an der Havanna-Konferenz vom September 1979 formuliert und ist auf der Idee begrUndet, dass der zweite grosse Schub der Erdß̈lpreiserhöhungen die Industrielander ernsthaft an den Verhandlungstisch bewegen wörde. Ferner hofften die Initianten, dass die Gruppe der 77 sich diese zweite Gelegenheit für Globalverhandlungen mittels engerem Schulterschluss und besserer Organisation nicht entgehen lassen würden. Die politische Grundvoraussetzung sollte wiederum dieselbe sein : Erdbldialog mit den Industrielandern im Austausch für Erfüllung 
von Forderungen der dritten Welt im Zusammenhang mit dem globalen Strukturwandel der neuen Weltwirtschaftsordnung. Zum Unterschied zur Situation vor fünf Jahren bestand aber nicht mehr die Sicherheit, ob alle OPEC-Staaten - und unter ihnen insbesondere Saudiarabien - die neue blockfreie Front voll unterstb̈tzen würden.

Nach zähen Verhandlungen stimmte die 34. UNO-Generalversammlung einer Resolution zu (Dezember 1979), in welcher sich alle UNO-Mitglieder schliesslich zu einer neuen Runde von Globalverhandlungen verpflichteten. Diese Resolution 34/138 legte als Haupttraktanden Rohstoffe, Handel, Entwicklung, Wuhrungsordnung und Finanz, sowie Energie fest (wobei Energie zum ersten Mal als Traktandum einer UNO-Globalverhandlung figuriert) und ordnete an, dass die entsprechenden Verhandlungen "integriert" und "konkret" gefuhrt werden sollen. Ein bereits bestehender Plenarausschuss der Generalversammlung (comité plénier), dem Dank der "alleStaaten"-Formel (1) auch die Schweiz angehört, wurde beauftragt, die Modalitbten der Globalverhandlungen so vorzubereiten, dass daruber (zusammen mit der neuen UNO-Entwicklungsstrategie des dritten Dezennium) in der elften Sondersession der Generalversammlung (August/September 1980) Einigung erzielt werden konnte. Die neue Runde hätte dann im Herbst 1980 durch die 35. ordentliche Session der Generalversammlung formell lanciert werden und im Fröhahr 1981 ihren Anfang nehmen sollen.

Einigung ist jedoch bis zum Beginn der 36. ordentlichen Session der Generalversammlung (September 1981) immer noch nicht erzielt worden, und das Schicksal der neven Runde befindet sich heute immer noch in der Schwebe. Die Gründe dafür sind in diesem Abschnitt zusammengefasst.

Als erstes ist auf die Zielsetzung der Resolution hinzuweisen : Erstens ist sie auf die Umstrukturierung der internationalen Wirtschaftsbeziehungen ausgerichtet, um damit eine regelmussige, globale Wirtschaftsentwicklung zu ermöglichen. Dabei wird der beschleunigten Entwicklung der Entwicklungs/ander ein besonders grosses Gewicht eingerbumt. Zweitens werden alle Sektoren der gegenwärtigen Wirtschaftskrise gleichzeitig angesprochen, damit Uber die gesamte Problematik in integrierter Weise verhandelt werden kann. Drittens sollen die Hauptverhandlungen auf der Ebene der UNOGeneralversammlung stattfinden, damit ein tragbares Gleichgewicht 
zwischen den Problemen und Vorschlagen in den einzelnen Sektoren und den verschiedenen technischen Gremien des UNO-Systemes hergestellt werden kann. Viertens soll dieser Verhandlungsansatz sowohl für konjunkturelle als auch für strukturelle Massnahmen gelten. Damit würde im Rahmen der UNO die Mugglichkeit des globalen Krisenmanagement geschaffen werden - und zwar in einer Weise, die einen Fortschritt des längerfristigen Strukturwandels im Sinne der neuen Weltwirtschaftsordnung ermbglicht.

Diese in der Resolution enthaltene Definition der Globalitat galt es zu konkretisieren und in ein realisierbares Verhandlungsschema zu Ubersetzen. Diese Arbeit ist jedoch aus drei Gründen seit der elften Sondersession der Generalversammlung (September 1980) steckengeblieben : wegen Verfahrensfragen, wegen der Tagesordnung, und wegen des Zeitplanes.

Verfahrensfragen : Eine Annuherung konnte auf informeller Ebene darÜber erzielt werden, dass die Globalverhandlungen in drei Phasen stattfinden sollen, (a) einer ersten konzeptionnellen Phase, in welcher auf der zentralen Ebene der UNO-Generalversammlung die Grundsattze und Richtlinien festgelegt werden, (b) einer zweiten Phase, in welcher die einzelnen Verhandlungselemente auf die UNOSpezialorganisationen (FAO, WHO, UNESCO, ILO, Welt Bank / Wuhrungsfonds, etc.) und die UNO-Organe (wie UNCTAD, UNDP, UNIDO) aufgeteilt und dort im Einzelnen durchdiskutiert werden; dabei müsste für die Verhandlung der Energiefragen eigens eine ad-hoc-Konferenz einberufen werden, weil weder ein UNO-Organ noch eine Spezialorganisation die Kompetenz daruber verfügt; und (c) eine dritte, wiederum zentrale Phase, in welcher die durchverhandelten Elemente und Entscheide zu einem integrierten Verhandlungspaket im Sinne der Resolution 34/138 zusammengefügt werden, uber dessen Ausführung sich alle Staaten einigen müssten. Dabei stünde auch eine Ueberprüfung der Resultate der letzten, einigermassen erfolgreichen UNO-Konferenzen Uber erneuerbare Energiequellen (August 1981) und Uber die Armsten Entwicklungslünder (September 1981) zur Diskussion. In dieser dritten Phase sollte sich insbesondere herausstellen, ob alle Elemente der Globalverhandlungen zusammenpassen, oder ob einzelne Punkte der zweiten Phase angesichts des Gesamtbildes neuverhandelt werden müssen.

Die Diskussion Uber diese Verfahrensfrage hat bis anhin den grossten 
Teil der Verhandlungszeit beansprucht. Doch ist bis heute keine Lbsung in Reichweite. Die Hauptschwierigkeit besteht in der Bedeutung der zweiten und der dritten Phase. Soll es in der Kompetenz der zentralen Konferenz stehen, in welcher alle Mitgliedstaaten gleiches Stimmrecht haben, die in der zweiten Phase getroffenen Vereinbarungen und Entscheide zu undern, wenn sie den Erfordernissen des angestrebten "integrierten Verhandlungspaketes" nicht genUgen? Haupthindernis bildet das Traktandum der Wührungsordnung, das heisst der Internationale Wahrungsfonds, dessen Struktur des gewichteten Stimmrechtes den westlichen Industrielundern eine automatische Mehrheit und den USA sogar ein faktisches Veto einraumt. Sollten die im IWF im Rahmen der Globalverhandlungen getroffenen Entscheide in der dritten Phase wieder in Frage gestellt oder gar abgeändert werden ?

Die USA, die keine Zweifel am status-quo des IWF zu erlauben gewillt waren, erhoben das Problem zu einer Frage des Prinzipes und widersetzten sich dem vorgeschlagenen Verfahren mit der Begründung, die Globalverhandlungen dürften keine Rivalitüten oder gar Kompetenzkonflikte innerhalb des UNO-Systemes heraufbeschwbren. Die Bundesrepublik Deutschland und Grossbritannien gesellten sich zu dieser Opposition, welche unter dem Uebernamen "Dreierbande" die Vorbereitungen der Globalverhandlungen schliesslich zum Stillstand brachte.

Der Präsident der 35. Generalversammlung vermochte diese Bescherung bis zum Dezember 1980 nicht in Ordnung zu bringen. Als Vermittler hat er sich jedoch durch das ganze Fröhiahr und den Sommer 1981 hindurch bemUht, auf dem Weg informeller diplomatischer Konsultationen Uber die Verfahrensformel Einigung zu erzielen, und hat wiederholt erwahnt, "90\% der Schwierigkeiten" seien aus dem Weg gertumt.

Nach dem Machtwechsel in den USA und den infolge des massiven Rechtsrutsches entstandenen schlimmen Befürchtungen Uber die Zukunft der Entwicklungszusammenarbeit, stellte sich nach der Verbffentlichung des Schlusscommuniqués des Ottawas-Gipfels der sieben wichtigsten westlichen Industrielunder (Juli 1981) gleichwohl ein milder Optimismus für die Globalverhandlungen ein. Alle Augen sind nun auf den Nord-SUd-Gipfel von 22 Staaten, einschliess der USA, in Cancun, Mexico, gerichtet (Oktober 1981), von dem ein 
neves Startzeichen erhofft wird.

Tagesordnung : Ueber die Tagesordnung der Globalverhandlungen und ihre verschiedenen Phases herrscht auch noch nicht grosse Klarheit. Verstandlicherweise haben die LOsungsversuche betreffend der Verfahrensfragen gegenÜber den Einzelheiten der Verhandlungsgegenstände den Vorrang eingenommen. Allerdings ist zu bemerken, dass abgesehen von den in der Resolution 34/138 vorgesehenen fünf "klassischen" Themen bis heute noch wenig Ober die konkrete Zielsetzung der Globalverhandlungen bekannt ist.

Die Hauptschwierigkeit mit der Tagesordnung besteht nach wie vor im Zusammenhang mit den Wührungs- und Energie(= Erdul)fragen . Die Entwicklungslander verlangen bekanntlich tiefgreifende und grundsatzliche Reformen im internationalen Währungssystem, die schon mindestens seit 1974 auf intergouvernementaler Ebene bekannt sind, und Uber die im Rahmen des IWF gelegentlich sogar schon Konsensus erzielt worden war, ohne dass jedoch zu deren Durchführung geschritten worden wäre; diese schliessen ein : bessere und gerechtere Anpassung der Reservebildung an die Wachstumsbed Urfnisse der Weltwirtschaft, insbesondere an diejenigen der EntwicklungsIander; Anpassungsdruck nicht nur gegenüber Defizit-, sondern auch gegenÜber Ueberschusslundern; weitere Verbreitung der IWF-Sonderziehungsrechte als Reserveeinheiten; Verbindung der Schaffung von Sonderziehungsrechten mit Entwicklungsfinanz; gerechtere Verteilung der globalen Kontrolle Uber das internationale Währungssystem. Die Industrieländer, unter der Führung der USA und der Bundesrepublik Deutschland, stellen sich auf den Standpunkt, dass Aenderungen im internationalen Wahrungssystem nur sehr langsam erfolgen dürfen, innerhalb des IWF auch tats blchlich unternommen würden, und dass es unklug wäre, diesen Prozess von aussen her zu unterbrechen.

Der Wahhrungsblockade auf der Seite der Industrieländer entspricht die Harte der OPEC-Staaten in der Energiefrage. Die Industrieländer erwarten, dass uber Voraussehbarkeit der Versorgung und der Preisbildung im Erdblbereich verhandelt werden wird und darlber konkrete Resultate erzielt werden. Die Losung dieser Fragen war zum vorneherein entscheidend für ihr Interesse an den Globalverhandlungen. Die OPEC-Staaten haben aber bis heute noch nicht zu erkennen gegeben, ob sie tatsächlich Uber Preise und Versorgung 
zu verhandeln bereit sein werden. Sie unterstreichen die Komplexitat solcher Verhandlungen und weisen darauf hin, dass es bis anhin nicht einmal innerhalb der OPEC moglich war, befriedigende Einigung Uber Produktion und Preise zu finden. Im Ubrigen bestehe kein zwingender Grund, die Erdלlpreise zu regulieren, solange es die Industrielunder ablehnen, eine entsprechende Regulierung ihrer Exportglbter in Erwägung zu ziehen.

Bezüglich der OPEC-Beziehungen mit der Übrigen dritten Welt steht es allerdings anders. Wahrend die Industrielander immer noch zu Uberzeugen sind, dass ihre WBnsche betreffend Versorgung und Preise nur durch entsprechend wichtige Konzessionen auf anderen Gebieten eingehandelt werden k8nnen, haben die OPEC-Staaten schon in ihrer Caracas-Konferenz (1979) Entwicklungslandern eine Versorgungsgarantie zu Vorzugspreisen zugebilligt.

Infolge der erwähnten Verfahrensschwierigkeiten wurde das Problem der Tagesordnung bis zum Dezember 1980 gar nicht richtig durchberaten, und blieb offen. Im Verlauf des Frühahres 1981 hat der Prasident der Generalversammlung jedoch mehrmals angetbnt, dass "Umstrukturierungen" der Tagesordnung informell im Gange seien, uber deren Natur und Tragweite allerdings noch nichts bekannt sind.

Zeitplan : Die 35. Generalversammlung hattte beschliessen sollen, die Globalverhandlungen im Frßhjahr 1981 zu beginnen. Um angesichts der erheblichen Schwierigkeiten die Globalverhandlungen zu retten, wurde die 35. Generalversammlung im Dezember 1980 nicht geschlossen, sondern bloss vertagt, in der Hoffnung, nach der Einigung Uber die Verfahrens- und Tagesordnungsfragen noch vor der Erbffnung der 36. Generalversammlung (September 1981) einen neuen Zeitplan festzulegen. Diese Hoffnung hat sich ebenfalls nicht verwirklicht. Ein weiteres ungelystes Problem ist die vorgeschlagene Lange der ersten, konzeptionellen Phase der Globalverhandlungen. Vorgesehen ist eine Dauer von acht Wochen, nach welcher die sektoriellen Verhandlungen der zweiten Phase beginnen sollten. Einige Industrielunder (unter ihnen die Schweiz), die aus der Ziellosigkeit der Pariser-KIWZ Lehren ziehen mbchten, finden diese acht Wochen zu kurz und tendieren auf eine "realistischere", längere erste Phase. 
Die Schweiz ist an den jüngsten Versuchen, die Vorbereitungen der Globalverhandlungen zu deblockieren, nicht offiziell beteiligt. Als Nicht-Mitglied der UNO konnte sie weder in den Verhandlungen der elften Sondersession noch in den ordentlichen Sessionen der UNO-Generalversammlung eingreifen. Der Ottawa-Gipfel war ohnehin Sache der westlichen grossen Sieben, und beim CancunGipfel, durch Oesterreich und Mexico als Nachfolgeereignis der Brandt-Kommission organisiert, bleibt diesmal die Schweiz draussen.

Die Verantwortlichen Bundesstellen, Schweizer OECD- und UNODelegationen, haben jedoch im Rahmen der Mitgliedschaft der Schweiz bei der UNO-Plenarkommission (comité plénier) bis zum Zeitpunkt der elften Sondersession der Generalversammlung einen bemerkenswerten und konstruktiven Beitrag geleistet.

Der Beitrag der Schweiz betrifft die Konzeption der Globalverhandlungen und die Struktur der Tagesordnung. Um die Sackgasse in bezug auf die Tagesordnung zu vermeiden, schlug die Schweiz im Gegensatz zum sektoriellen Ansatz (Rohstoffe, Handel, Entwicklun Wuhrungsordnung und Finanz, sowie Energie) - einen nach den wichtigsten Gegenwartsproblemen gerichteten thematischen Ansatz vor. Als Beispiele solcher Themen wurden die Zahlungsbilanzproblematik, die Ernthrung und Landwirtschaft sowie die Energie erwähnt.

Der thematische Ansatz bietet die M४glichkeit einer Konzentration auf spezifische Gegenwartssituationen sowie die Mbglichkeit einer realistischen Zielsetzung und raumt der zentralen Konferenz (erste und dritte Phase) die Funktion ein, auf Sektorenebene vorgeschlagene Massnahmen nach den grossen Themen und deren Zielsetzung zu ordnen und die notwendigen Querverbindungen zwischen den verschiedenen sektoriellen Problemen herzustellen. Dem umstrittenen zentralen Verhandlungsorgan wäre damit eine nach Themen konzipierte Koordinationsrolle gegeben. Die Doppelspurigkeit und der Kompetenzkonflikt um das Wiederverhandeln von schon Verhandeltem konnten dabei vermieden werden. Als wichtiges Element wurde die konkrete Zielsetzung hervorgehoben, deren Zweck es war, einen Verhandlungsfortschritt gegenuber den früheren Globalkonferenzen zu 
ermøglichen.

Die Struktur der thematischen Konzeption wurde am Zahlungsbilanzproblem vorgefuhrt :

\section{THEMA : ZAHLUNGSBILANZPROBLEME}

ZIEL 1 : Stabilisierung und Voraussehbarkeit der Exporteinnahmen der Entwicklungsländer.

Massnahmen betreffend :

- Rohstoffhandel

- Protektionismus

- Finanzierung von Kompensationen für Entwicklungslander.

ZIEL 2 : Vergrobserung der Exporteinnahmen der Entwicklungslander.

Massnahmen betreffend :

- Verarbeitung von Rohstoffen

- Ausbeutung von neuen Energiequellen, einschlisslich Zugang zu und Finanzierung von entsprechender Technologie

- Zugang zu den Mürkten der Industrielünder und zu Exportkrediten

- Handel unter den Entwicklungslander.

ZIEL 3 : Vergrosserung der finanziellen Unterstötzung für Entwicklungsländer (insbesondere für die Ärmsten Lünder) zu vergünstigten - sowie zu Martkbedingungen.

Massnahmen betreffend :

- Rolle, Verwendung und Volumen der bffentlichen Entwicklungshilfe

- privatwirtschaftliche Kapitaltransfers und Risikoverteilung

- andere finanzielle Massnahmen, Technologietransfer, Verschuldung.

Eine analoge Themen-, Ziel- und Massnahmenordnung künnte für die anderen grossen Themen erarbeitet werden. Dabei ware es Sache der Vorbereitungen und der ersten Phase der Globalverhandlungen, Einigung Uber einen ausgewogenen Themenkatalog zu erzielen und die Zielsetzung der einzelnen Themen festzulegen. Damit watre den darauffolgenden sektoriellen Verhandlungen (zweite Phase) ein realisierbarer, globaler Rahmen gegeben. 
Im Gegensatz zu den Industrielandern, welche die Vorbereitung der Globalverhandlungen schliesslich zum Stillstand brachten, anerkannt, die Schweiz das UngenÜgen der bisherigen Verhandlungen auf der sektoriellen Ebene der verschiedenen UNO-Organe und -Organisationen und schloss sich der Forderung der Gruppe der 77 nach einer zentralen Verhandlungsorgan an. Den Themen und Meinungen, die an der UNO-Generalversammlung diskutiert und verhandelt werden können, sollten keine starren Grenzen gesetzt werden. Doch sollte das zentrale Organ der dritten Phase eine beurteilende und bewertende, nicht aber eine abändernde Funktion haben. Die Forderung nach einer abandernden Funktion wäre nach schweizerischer Meinun juristisch ohnehin nicht haltbar.

Es ist durchhaus mbglich, dass die Frage der Tagesordnung in der Richtung der schweizerischen Idee eine Losung finden wird. In der Tat war der Schweizer Vorschlag bei den OECD-Staaten, insbesondere bei der Europdischen Gemeinschaft, auf Interesse gestossen und hat die gemeinsamen Vorbereitungen der westlichen Industrielunder erheblich beeinflusst. Allerdings enthalt dieser Vorschlag noch keinen Ansatz zur integralen Behandlung der zentralen Fragen der inte nationalen Wahrungs- und Finanzordnung sowie des Erdbls, auf welchen die Gruppe der 77, insbesondere die OPEC-Staaten, eintreten könnten.

\section{NACH OTTAWA UND CANCUN : WAS TUN ?}

Von den 38 Paragraphen der Ottawa-Erklärung (Juli 1981) sind 13 den "Beziehungen zu den Entwicklungslandern" gewidmet. Diese enthalten Hinweise auf die wirtschaftliche Interdependenz sowie auf das gemeinsame Interesse von SÜd und Nord an der Entwicklung der dritten Welt; Feststellungen ober die "schreckliche Armut"; eine Beteuerung, die Bffentliche Entwicklungshilfe "substantiell" zu erhלthen, den Strom privaten Kapitals in die Entwicklungsländer zu erhöhen (sofern diese genügend Zusicherungen für dessen Schutz und Sicherheit geben); Forderungen an die Erdbl- und Ostblockländer, mehr zu tun; Besorgnisse uber das Bevolkerungswachstum und Erkenntnis der Bedeutung weltweiter Sicherheit der Nahrungs- 
mittelversorgung.

Zwei Paragraphen sind den Globalverhandlungen gewidmet :

"11. Wir sehen konstruktiven und substantiellen Erörterungen mit Entwicklungsländern entgegen und sind der Auffassung, dass das Gipfeltreffen in Cancun (Mexico) eine baldige Gelegenheit bietet, unsere gemeinsamen Probleme erneut zu behandeln."

"12. Wir bekruftigen erneut unsere Bereitschaft, alle M४glichkeiten der Konsultation und Zusammenarbeit mit Entwicklungslandern in jedem hierfur geeigneten Forum zu erkunden. Wir sind bereit, an der Vorbereitung für einen beiderseitig akzeptablen Prozess globaler Verhandlungen mitzuwirken, die Aussicht auf sinnvollen Fortschritt bieten."

Erklart wurde also die Bereitschaft "zu erkunden", zur Mitarbeit "an der Vorbereitung", nicht aber unbedingt an Globalverhandlungen; im Ubrigen bleiben die UNO-Resolution 34/138 und die langen Monate zöhen Verhandelns und Vorbereitens unerwähnt.

In Ottawa mag vor allem der kanadische Ministerprasident dem neven Präsidenten der USA diesen kollektiven Schritt in Richtung einer "Vorbereitung" abgerungen haben. Kommentatoren haben infolgedessen von einem "Fortschritt" oder gar einem "Durchbruch" geredet. Vom Standpunkt der dritten Welt, insbesondere der Gruppe der 77, muss die Ottawa-Erklarung jedoch als ein Rückschritt auf den Nullpunkt, das heisst auf die Ausgangslage vor der Resolution 34/ 138 (1979), interpretiert werden. Zum Trost soll aber der CancunGipfel den "Vorbereitungen" erneut zu einem Profil verhelfen.

Die neve Lage ist mit der langsamen, neven Stellungnahme der USA zur Entwicklungsproblematik verbunden. Die Entwicklungszusammenarbeit scheint jetzt erstens wieder der amerikanischen Ost/West-Auseinandersetzung untergeordnet zu sein. Zweitens wird das Konzept des globalen Strukturwandels und die von allen UNOMitgliedern angenommene Resolution uber die neue Weltwirtschaftsordnung (1975) deutlich abgelehnt. Drittens kommt eine globale Umverteilungspolitik zu Gunsten der dritten Welt, einschliesslich massive Transfers von 8 ffentlichen Mitteln der Industrielander, nicht mehr in Frage. Viertens soll die ohnehin schon deflationare Kreditpolitik des IWF verschürft werden, und fünftens soll die Welt Bank, 
die in amerikanischen Regierungskreisen gelegentlich sogar als "sozialistisch" bezeichnet wird, in Zukunft weniger auf uffentliche, dafür vermehrt auf private (das heisst : teurere) Finanzmittel zühlen müssen.

Die Vorzeichen des Cancun-Gipfels (Oktober 1981) sind zwar insofern positiv, als er Uberhaupt stattfinden wird, allerdings inmitten des amerikanischen Versuches, zehn Jahre globale Entwicklungspolitik zu ignorieren und/oder wieder rückgüngig zu machen.

Den eingeladenen Staatsoberhđuptern (2) wird keine Tagesordnung vorgelegt werden; die Gipfel-Ambiance soll so informell wie nur möglich gehalten werden. Auf der Ebene der Aussenminister ist allerdings ein Gesprbchsrahmen vorgeschlagen worden (August 1981), welcher die Aufmerksamkeit des Gipfeltreffens auf die Zukunft der internationalen Entwicklungszusammenarbeit und die Wiederbelebung der Weltwirtschaft zu richten versucht. Dabei werden besonders die Gebiete der Ernuhhrung, der landwirtschaftlichen Entwicklung, der Rohstoffe, der Industrie und des Handels, der Energie sowie der Wahrungs- und Finanzfragen erwahnt. Obwohl keine formelle Verbindung zu den entsprechenden Anstrengungen an der UNO besteht, wird erwartet, dass einer Form von Globalverhandlungen das gröne Licht gegeben wird, wobei im besten Fall der Schritt vom OttawaZugeständnis der "Vorbereitung" zu einer gemeinsamen Verpflichtuns zu "realistischen" Globalverhandlungen erwartet wird. "Realistisch" wird bedeuten, dass das UNO-Konzept mehr den Forderungen der harten Staaten angepasst werden muss. Wie weit diese Anpassung gehen wird, ist allerdings unsicher und wird in Cancun wohl kaum beschlossen werden. Es ist dabei zu bedenken, dass die Gruppe der 77 ihre Organisation und ihren inneren Zusammenhalt zu verstarken im Begriffe ist, wurde doch an der Caracas-Konferenz uber wirtschaftliche Zusammenarbeit unter Entwicklungsländern (ECDC, Mai 1981) die Errichtung eines gemeinsamen Arbeitsmechanismus und Verhandlungssekretariates beschlossen.

Wenig berUhrt von den weltweiten Anstrengungen, den Nord-SUdDialog zu erneuern, verbreitet sich in der offiziellen Schweiz seit einiger Zeit eine verstärkte Stimmung des RÜckzuges gegenÜber den Forderungen der dritten Welt. Das Budget der Bffentlichen Entwicklungszusammenarbeit ist um $18 \%$ gekürzt worden, mehr als der Durchschnitt der Kurzungen in anderen Bereichen. Der konservative 
Gedanke ist wieder modern und zeigt sich besonders nutzlich in der Ablehnung von Konzessionen gegenüber der dritten Welt. Obwohl die politische und wirtschaftliche Konjunktur heute denkbar ungünstig und unfreundlich ist, stellen wir zum Abschluss gleichwohl noch eine Frage und versuchen ein paar Antworten, die dem heutigen Realpolitiker "unrealistisch" erscheinen mbgen, die jedoch etwas mit der Zukunft von uber 800 Millionen unter der Armutsgrenze lebenden Menschen zu tun hat.

Welcher Art wären einige positive Geitrlge, welche die Schweiz in den kUnftigen Globalverhandlungen leisten könnte?

Die Schweiz kann ihr Engagement und Interesse auf verschiedenen Gebieten der Globalverhandlungen verstärken und ausbauen; ein gewisses Verständnis dazu besteht bereits in der Bundesverwaltung. Die Weltwirtschaft ist neven politischen und zkonomischen Verhältnissen anzupassen. Dabei hat die Schweiz infolge ihrer grossen wirtschaftlichen Abhangigkeit vom Ausland und ihrer kommerziellen und finanziellen VerknUpfung mit verschiedenen Teilen der dritten Welt ein besonderes Interesse an geordneten internationalen Verhultnissen. Globalverhandlungen Uber die Zukunft der Weltwirtschaft und der Entwicklungszusammenarbeit, ob nach dem UNO-Konzept oder in einer modifizierten Form, sind infolgedessen wichtig für die Schweiz. Die Schweiz sollte an jeder zukunftigen Form von Globalverhandlungen konstruktiv mitarbeiten, wobei einem universellen Forum der Vorzug gegeben werden sollte.

Die Schweiz hat ein vorzügliches Gesetz Uber die Entwicklungszusammenarbeit, das auf die Grundbedß̈rfnisse lokaler Bevollkerungen und auf den Ausbau ihrer Eigenstandigkeit ausgerichtet ist. Das Gesetz sollte nicht unter dem Drang zur "Anpassung an neve Verhältnisse" verwässert werden.

Die Uffentlichen Mittel der Entwicklungszusammenarbeit waren bekanntlich schon vor den neuesten Kürzungen äusserst bescheiden und bewegten sich um 0,2\% des Bruttosozialproduktes; nur Italien und Oesterreich (und neverdings wahrscheinlich auch die USA) haben weniger getan. Es steht dem Land mit dem hblchsten proKopf-Einkommen der Welt zu, mehr zu tun. Trotz der Finanzmisere des Bundes sollten die Bffentlichen Entwicklungsmittel nicht gek Brzt werden. Ein bescheidener Anfang kann damit gemacht werden, die in der Botschaft des Bundesrates vom 15. August 1980 in Aussicht 
gestellte, graduelle Anpassung der 8 ffentlichen Entwicklungshilfe an den Durchschnitt der OECD-Länder $(0,35 \%$ des Bruttosozialproduktes) beizubehalten. Andere europäische Lünder mit ühnlichen oder schwierigeren $8 f f e n t l i c h e n$ Finanzproblemen lassen bei ihren SparUbungen die Uffentliche Entwicklungshilfe aus wichtigen politischen Erwägungen unangetastet. In den Niederlanden wird eine Kurzung der Gehblter des Staatspersonals erwogen; die Entwicklungshilfe bleibt aber intakt. Italien erhbht seine 8 ffentliche Entwicklungshilfe substantiell, und Frankreich hat sogar eine reale Verdoppelung wathrend der Aera Mitterrand in Aussicht gestellt.

Es ist bekannt, dass der Mobilisierung von Entwicklungshilfemitteln durch den $8 \mathrm{ffentlichen} \mathrm{Haushalt} \mathrm{heute} \mathrm{in} \mathrm{allen} \mathrm{Industrieländern}$ Grenzen gesetzt sind. Anstrengungen sollten infolgedessen unternommen werden, zusätzliche Mittel auf anderen Wegen zu beschaffen; zum Beispiel :

Die Schweiz könnte als wichtiges Währungsland einen Beitrag leisten in der Vermittlung zwischen den legitimen Forderungen der dritten Welt auf dem Gebiet der internationalen Währungsreform und der Politik derienigen Lunder, welche die atlantische Wuhrungsblockade immer noch aufrechterhalten.

Eine weitere Mタglichkeit besteht darin, $8 \mathrm{ffentliche}$ und private Finanzmittel in einer für Entwicklungszwecke geeigneten Weise zu verbinden. GestÜtzł auf Risikoversicherung, Garantieen, Zinsverbilligungsmechanismen und einen geeigneten entwicklungspolitischen Rahmen könnte privates Investitionskapital vermehrt in Bahnen geleitet werden, die den einheimischen PIBnen und Leistungsmbglichkeiten der Entwicklungslander entsprechen. Viele europdische Staaten verfUgen heute Uber Institutionen, welche auf die Verbindung von uffentlicher und privater Entwicklungszusammenarbeit spezialisiert sind und einen erheblichen Kapitaltransfer vornehmen.

Ferner kßunnten verschiedene Vorschläge für mehr Automatizitat in der Entwicklungsfinanz, einschliesslich Formen der internationalen indirekten Besteuerung, wie diese von des Brandt-Kommission (internationale Umsatzstever) und der Dag-Hammarskjold-Stiftung (Petroleum- und Erdgasstever; monetare Massnahmen) vorgeschlagen wurden, geprüft und den massgebenden internationalen Gremien zur Bearbeitung Ubergeben werden. 
Auch auf aussenwirtschaftlichem Gebiet bestehen M४glichkeiten, zusatzliche Beiträge zu leisten. Die Schweiz hat bekanntlich einen grossen Handelsbilanzüberschuss mit den Lündern der dritten Welt - eine Tatsache, welche zur wachsenden Verschuldung dieser Lunder beitrlagt. Der Schweizer Konsument fordert aber Qualitatsprodukte und eine regelmussige Versorgung. Um Importe aus den Lündern der dritten Welt zu fördern, könnten die zustündigen Behßrden und Organisationen helfen, das Angebot aus der dritten Welt den schweizerischen Verhalltnissen anzupassen und Handelskontakte aller Art zwischen Exporteuren aus der dritten Welt und Schweizer Importeuren zu ermbglichen. Viele europutische Lunder sind auf diesem Gebiet bereits tatig, wobei die skandinavischen Lander und die Niederlande am meisten Erfahrung haben.

Ferner konnte die Schweiz, in engerer Zusammenarbeit mit fortschrittlichen europüischen Staaten, dazu beitragen, die Verhandlungen uber den Verhaltenskodex für transnationale Unternehmen und über den Technologietransfer sowie die Verhandlungen uber die internationale Patentrechtsrevision so voranzubringen, dass diese in einer den Entwicklungslündern nützlichen und deren Eigenständigkeit fürderlichen Weise abgeschlossen werden können.

Die Verschlechterung des Aussenhandels der Entwicklungsländer ist eng mit der Ueberwälzung steigender Kosten, insbesondere der Energiekosten, in den Industrielandern verbunden. Um dieser Tendenz entgegenzuwirken, werden in der Europdischen Gemeinschaft und im Rahmen des IWF finanzielle Massnahmen ergriffen, welche den Entwicklungslandern helfen, ihre Exporteinnahmen zu stabilisieren und die Preisverhallnisse ihres Aussenhandels zu verbessern. Als einziges westliches Industrieland kann sich die Schweiz um Beiträge zu solchen Massnahmen dröcken. Auch als Nicht-Mitglied sollte die Schweiz jedoch freiwillig und parallel mit den anderen Industrieländern entsprechende Beitrlage zu solchen internationalen handelspolitischen Instrumenten leisten.

Infolge des in der Schweiz herrschenden ordnungspolitischen Gedankengutes mag die Versuchung heute gross sein, die konservative Welle aus den USA zu begrussen und mit dem neuen DinosaurierLiberalismus gemeinsame Sache zu machen. Es wäre zu probfen, ob diesem Gedankengut, sowie der Wirtschaft und der internationalen Glaubwördigkeit, mehr gedient sein künnte, wenn gemeinsame Sache 
mit den entwicklungspolitischen Zielen der kleinen Staaten Europas und mit unseren Partnern in der dritten Welt gemacht wörde.

\section{NACHWORT UND LITERATUR}

Der Verfasser dankt vorallem Liaqat Ali, Gerassimos Arsenis, K.K.S. Dadzie, Lucien Erard, Charlotte Feller, Jacques Forster, Marc Nerfin, Peter Saladin und Michael Zammit-Cutajar für die Beantwortung vieler Fragen. Die Verantwortung für die Interpretation dieser Gespräche und für alles Geschriebene ubernimmt er jedoch selber. Im Zusammenhang mit der Vorgeschichte, der ersten Runde und dem darauffolgenden Stillstand des Nord-SÜd-Dialoges haben sich die folgenden Dokumente als nützlich erwiesen :

Karl P. Sauvant - The Group of 77 : Evolution, Structure, Organization. New York : Oceana, 1981.

Karl P. Sauvant und Hajo Hasenpflug (eds.) - The New International Economic Order : Confrontation or Cooperation between North and South? Boulder : Westview Press, 1977.

"The Stockholm Declaration" - Stockholm, Sweden, 5-16 June 1972. In : In Defence of the Earth. Nairobi : UNEP Executive Series, 1981.

Arjun Sengupta - "A review of the North-South negotiating process", IFDA Dossier 18, July/August 1980.

Arjun Sengupta (ed.) - Commodities, Finance and Trade : Issues in North-South Negotiations. London : Frances Pinter, 1980.

Conference on International Economic Cooperation, Ministerial Conferences. Paris, 6-19 December 1975, Paris, 30 May2 June 1977 (final document, Paris, 1977).

Im Zusammenhang mit dem neven Ansatz :

Anthony Dolman und Jan van Ettinger (eds.) - Partners in Tomorrow. New York : Dutton, 1978. 
Nations Unies, Comité plénier - Document de travail présenté par la Suisse. New York, A/AC.191/1/1980/CRP.4, 3 avril 1980.

United Nations - Iowards the new international economic order : analytical report on developments in the field of international economic cooperation since the sixth special session of the General Assembly. New York, A/S-11/5, 7 August 1980. Global Negotiations Action Notes. New York : UN Church Centre, Nr.1-19, Dezember 1980 bis August 1981.

International Foundation for Development Alternatives - Special United Nations Service. Nyon : Nr. 198-354, 5. Januar 1981 bis 31. August 1981.

"Erklürung des Gipfeltreffens von Ottawa", Neve Zürcher Zeitung,

Nr. 169, 23. Juli 1981 .

Ferner auch :

Botschaft des Bundesrates Uber die Weiterführung der technischen Zusammenarbeit und der Finanzhilfe. 15. August 1980.

North-South: a programme for survival. The Brandt-Commission Report, London : Pan Books, 1980.

"Automatic mobilization of resources for development", Report of the Dag Hammarskjold Foundation, Development Dialogue, $1981: 1$.

\section{ANMERKUNGEN IM TEXT}

1. Es handelt sich um eine Klausel, durch welche "allen Staaten", und nicht bloss "allen UNO-Mitgliedern" das Recht der Mitarbeit an den Vorbereitungen der Globalverhandlungen eingerbumt wird.

2. Industrielander : Oesterreich und Kanada (Hauptinitianten), Frankreich, Bundesrepublik Deutschland, Schweden (für Skandinavien), Grossbritannien und USA; Entwicklungslander : Mexico (Hauptinitiant und Gastland) plus drei pro Region : Nigeria, Tanzania, ElfenbeinkUste; Bangladesh, Indien, 
Philippinen; Brasilien, Venezuela, Guyana; plus zwei für Arabien : Algerien, Saudi Arabien; plus China und Jugoslawien. 


\section{ANHANG : FORDERUNGEN DER DRITTEN WELT}

Ausschnitt und Zusammenfassung der im Rahmen der Vorbereitungen zur elften Sondersession der UNO-Generalversammlung von der Gruppe der 77 vorgeschlagenen Massnahmen.

\section{A. Internationaler Handel und Rohstoffe :}

Schaffung von besseren allgemeinen Handelsbedingungen, einschliesslich mehr Handelsvergünstigungen und weniger Handelsschranken, insbesondere Verhinderung von neven Handelsschranken;

Erneverung der Prinzipien und Regeln des internationalen Handels durch eine Internationale Handelkonferenz der UNO;

vollständige und prompte Ausführung der im Rahmen der TokyoRunde beschlossenen Abschaffung von nicht-tarifuren Handelsschranken ;

Abschaffung aller gegenUber der dritten Welt bestehenden Handelsschranken bis 1985;

multilaterale Vorsichtsmassnahmen gegenUber Marktunterbrllchen und -verzerrungen und Kompensation für Verlust von Exporteinnahmen; vollstandiges und wirkungsvolles Funktionieren des gemeinsamen Rohstoffonds;

Abschluss von mindestens zehn zentralen Rohstoffabkommen im Rahmen des intergrierten Programmes;

Verarbeiten der Rohstoffe in den Produzentenlundern sowie Teilnahme an der Vermarktung, Verteilung und Transport;

Zugang zu Murkten für landwirtschaftliche Produkte der Dritten Welt und Abschaffung der Handelsschranken gegenUber tropischen Produkten ;

Internationale Regelung der Herstellung von synthetischen Produkten und Verbesserung der Wettbewerbsfahigkeit von Naturprodukten; Verhandlungen Uber sektorspezifische Handelsfragen; 
Erweiterung des bestehenden allgemeinen Systemes der Handelspraferenzen ;

Verbesserung der Deviseneinnahmen und Verhindern des Devisenabflusses beim unsichtbaren Handel (Transport, Versicherung, Bankwesen, Tourismus);

beschleunigtes Wachstum des einheimischen Versicherungs- und RUckversicherungswesens ;

Uebereinkunft Uber den Verhaltenskodex der transnationalen Unternehmen; wirkungsvolles Eliminieren deren negative Auswirkungen und Fürdern deren Beiträge zu den Entwicklungszielen der GastIonder ;

Eliminieren von restriktiven Geschaftspraktiken; Einberufen einer UNO-Konferenz Uber dieses Thema.

\section{B. Industrialisierung :}

Strukturwandel in den Industrieländern, um die Industrialisierungsziele der Dritten Welt zu ermoglichen, einschliesslich der Umverteilung und Neuschaffung von Produktionsstatten, Transfer von nicht-obsoleter Technologie sowie Abschaffung der Industrieprotektion und -subventionierung in Industrielandern;

Erweiterung des multilateralen Konsultationssystems der UNIDO; Verstärkung der Entwicklungszusammenarbeit auf dem Gebiet der industriellen Entwicklung; Schaffung eines globalen Industrialisierungsfonds för die Dritte Welt mit anfanglich US\$15 Mia. pro Jahr ;

FOrderung effizienter arbeitsintensiver Industrievorhaben;

Beschaffung der technischen und finanziellen Mittel für die industrielle Entwicklungsdekade Afrika's (1980-90).

C. Nahrung und Landwirtschaft :

AusfUhrung des an der Weltkonferenz Uber Agrarreform (1979) angenommenen Aktionsplanes;

entsprechende Aufstockung der bilateralen und multilateralen Finanzmittel, besonders diejenigen des Internationalen Fonds för Landwirt- 
schaftsentwicklung (IFAD);

weite Verbreitung von angepasster, kostensparender landwirtschaftlicher Technologie;

Verbesserung der Düngemittel, Samen und Pestizide; Verhinderung von Ernteverlusten, Kontrolle der Heuschreckenplage und der afrikanischen Schlafkrankheit;

Errichtung eines verlasslichen, weltweiten Nahrungsmittelsicherheitssystemes; internationale Koordination der Reservehaltung; Abschluss eines neuen Weizenabkommens;

verbesserte Nahrungsmittelhilfe (mit Zielsetzungen für 1985, 1990); Erhbhung der Mittel des Welternםhrungsprogrammes (WFP);

Ausdehnung der IWF-Fazilitלlten auf die Finanzierung erhbhter Nahrungsmittelimporte der Dritten Welt;

verscharfte und koordinierte Massnahmen zur Bekämpfung des Hungers und der Unterernahrung;

Ueberwachung der Auswirkungen des Nahrungsmittelhandels auf die Nahrungsmittelproduktion in der Dritten Welt.

D. Entwicklungsfinanz und Verschuldung :

Errichtung eines neven, umfassenden Systemes der internationalen Entwicklungsfinanz zur Unterstötzung der verstärkten Eigenanstrengungen der Entwicklungsländer;

vermehrte Verlässlichkeit, Kontinuitat und Automatizitat der Entwicklungsfinanz;

Verpflichtung der Industrielander bis zum Jahre $19820,7 \%$ des Bruttosozialproduktes (netto) an bffentlicher Entwicklungshilfe zu leisten und diese Leistung bis 1990 auf $1 \%$ des BSP zu erhbhen; Verdreifachung der realen 8 ffentlichen Entwicklungshilfe an die armsten Lunder bis zum Jahr 1985, und Vervierfachung bis zum Jahr 1990;

Verbesserung der Bedingungen der Bffentlichen Entwicklungshilfe; Verlängerung der Garantieperioden der Exportrisikoversicherungen der Industrielünder ; 
Verbesserung der Bedingungen und Vergrösserung der Reserven der multilateralen Finanzinstitute (Weltbank, IDA, regionale Entwicklung: banken), einschliesslich vermehrte Gewichtsverlagerung von der Projekt auf die Programmilfe; ;

Verbesserung der Bedingungen und Verbesserung des Entwicklungseffektes der privatwirtschaftlichen Finanzstrolme; Schaffung von offentlichen Garantieen und Zinssubventionen, um dies zu ermöglichen;

Liberalisierung der Kapitalmärkte der Industrieländer zu Gunsten der Entwicklungslünder;

Schaffung einer Fazilitbt zur Konvertierung von kurzfristigen Geldmarktkrediten in mittel- und langfristige Entwicklungsfinanz;

schnellstmbgliche Schaffung eines Notstandprogrammes von US $\$$ 4 Mia. jthrlich (zu vergünstigten Bedingungen) zu Gunsten der armsten Länder;

Beschleunigung der Verhandlungen Uber internationale Grundsätze und Richtlinien der Schuldensanierung nach den allgemeinen Grundsützen der UNCTAD;

Massnahmen zur Schuldenerleichterung, insbesondere zu Gunsten der armsten Länder, sowie retroaktive Anpassung der Schuldenlast der offentlichen Entwicklungshilfe an die geforderten, verbesserten Bedingungen und Gewährung einer gewissen Automatizitat der Schuldenanpassung an die Verschlechterung der weltwirtschaftlichen Lage;

Erleichterung der durch steigende Preise lebenswichtiger Importe bedingten, zusttzlichen Schuldenlast durch neve ZahlungsbilanzunterstÜtzungsmassnahmen des IWF und anderer zuständiger Organisationen ;

graduelle KÜrzung der Militärbudgets aller Länder, insbesondere solcher die Ober Nuklearwaffen verfögen und Umverteilung dieser Mittel auf soziale und wirtschaftliche Entwicklungsprogramme, insbesondere in der Dritten Welt.

E. Reform des internationalen Wahrungssystemes :

Aufbau eines internationalen Wührungssystemes, welches einen wirkungsvollen, fairen und symetrischen Anpassungsprozess mit 
hohen Wirtschaftswachstumsraten, hohem Beschaftigungsgrad und dynamischer Expansion des Welthandels ermbglicht;

Zugang der Entwicklungsländer zu uffentlichen Kreditfazilitüten, welche deren speziellen Zahlungsbilanzproblemen angepasst ist; vermehrter Zugang der Dritten Welt an die Finanz- und Kapitalmärkte der Industrielünder;

Revision der bestehenden IWF-Fazilitäten, um den Entwicklungsländern das Durchstehen der sich verschlechternen weltwirtschaftlichen

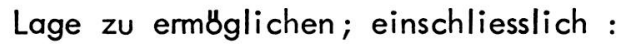

- Verlangerung der RUckzahlungsfristen,

- Aenderung der quantitativen Beschrönkungen bei bestehenden Fazilitaten,

- Aenderung der IWF-Entschadigungsfazilitל̈t (compensatory financing facility) durch Vergrbsserung des Volumens und Erleichterung des Zugangs, um Entwicklungslander genÜgend für die Verschlechterung der Preisverhaltnisse ihres Aussenhandels zu kompensieren;

Errichtung einer mittelfristigen Zahlungsbilanzfazilitltt im Rahmen des IWF mit Zinsverbilligung und einem Minimum an Konditionalitatt, entsprechen den Anpassungsbedingungen und -mø̈glichkeiten der Entwicklungsländer;

Massnahmen zur Ausgleichung der zunehmend ungünstigen Bedingungen privatwirtschaftlicher Finanzquellen;

Einführung eines Wechselkursrégimes, das - obwohl flexibel genUgend Wahrungsstabilitat zu fördern im Stande ist;

Schutz des realen Wertes der Exporteinnahmen der EntwicklungsItunder;

Gemeinsame Schaffung von internationaler Liquidität entsprechend den Bedürfnissen eines wachsenden Welthandels, mit spezieller RUcksicht auf die Probleme der Dritten Welt;

Erhebung der IWF-Sonderziehungsrechte zu Hauptreserven im internationalen Wahrungssystem und jahhrliche, den Notwendigkeiten der IWF-Mitglieder angepasste Schbpfung von Sonderziehungsrechten ;

Fürderung des Nettostromes von realen Resourcen von Industrielundern an die Entwicklungslunder durch Verbindung von Sonder 
ziehungsrechten mit Entwicklungsfinanz (SDR-link);

vergrbsserte Rolle der Entwicklungslander im Entscheidungsprozess des IWF und der Welt Bank.

\section{E. Energie :}

Sparmassnahmen im Energieverbrauch in allen Lündern, besonders aber in den Industrielandern;

Erforschung und Ausbeutung neuer konventioneller sowie alternativer Energiequellen im Rahmen der nationalen Entwicklungspläne und -prioritaten, und unter Mithilfe der internationalen Gemeinschaft, im Einklang mit der vollen und permanenten Souveranitat jedes Landes Ober seine Bodenschätze;

vermehrte Teilnahme an der Produktion, Verarbeitung, Vermarktung und der Verteilung solcher Bodenschatze;

Zusicherung unbeschrankten Zuganges zu wissenschaftlichen und technologischen Verfahren, einschliesslich Nukleartechnologie, durch die Industrielunder, um die Entwicklung und Produktion einheimischer Energiequellen zu ermöglichen und zu furdern;

mittel- und langfristige Aktionsprogramme för erneuerbare Energiequellen, einschliesslich Zusammenarbeit von Ländern mit uhnlichen geophysikalischen und klimatischen Bedingungen, in der Erforschung und Prufung solcher Energiequellen und bei der dabei notwendigen Ausbildung von Fachleuten;

susätzliche und besondere Massnahmen :

- zur Befriedigung der wachsenden EnergiebedUrfnisse der Entwicklungslander (Prioritat),

- zur Finanzierung und technischen Unterstotzung der Entwicklung aller Energiequellen in Entwicklungslandern,

- zur Erforschung und Entwicklung von neven einheimischen Energiequellen,

- zum Schutz und zur Verbesserung der Kaufkraft der Energieexporte der Produzentenländer der Driten Welt. 
Anmerkung

Weitere Massnahmen wurden auf den Gebieten der Wissenschaft und Technologie, des Transportwesens, der wirtschaftlichen Zusammenarbeit unter Entwicklungslander, des Umweltschutzes, der sozialen Entwicklungsprobleme sowie för die Brmsten Entwicklungslander, die Inselstaaten, die vom Land eingeschlossenen Entwicklungslander, und die von der Wirtschaftskrise am meisten betroffenen Staaten der Dritten Welt gefordert.

Quelle

"Synoptic table on the policy measures of the new international development strategy : informal proposal by the Groupe of 77", New York : United Nations (working paper 80-10169, 24 April 1980). 